\title{
Bloqueo paraespinal lumbar en pacientes con sindrome postlaminectomia.
}

\author{
Lumbar paraspinal block in patients with post laminectomy syndrome. \\ Gladys Pasco ${ }^{\text {1,a;2, }}$, Juana Arias Ramirez ${ }^{3, a}$

\section{RESUMEN}

Objetivo: Evaluar el beneficio del bloqueo para espinal lumbar en el tratamiento del Síndrome Post Laminectomía frente a la aplicación de agentes físicos. Material y métodos: Se realizó un estudio randomizado en 40 pacientes, de los cuales 20 recibieron bloqueo para espinal más terapia física y 20 sólo terapia física. Hemos evaluado la intensidad del dolor antes y después de la aplicación de cada terapia mediante la escala visual análoga (EVA). Resultados: El bloqueo para espinal lumbar fue exitoso en 18 pacientes, los otros 2 no mostraron mayor cambio con el tratamiento. Conclusiones: El bloqueo para espinal lumbar puede reducir el dolor y mejorar el estado funcional de los pacientes con síndrome post-laminectomía, permitiendo una mejor calidad de vida.

PALABRAS CLAVE: Laminectomía ; bloqueo neuromuscular.

\section{SUMMARY}

Objective: To evaluate the benefit of lumbar spinal block in the treatment of Post Laminectomy Syndrome compared to the application of physical agents. Material and methods: A randomized study was conducted in 40 patients, of whom 20 received spinal block plus physical therapy and 20 only physical therapy. We have evaluated the intensity of pain before and after the application of each therapy using the visual analog scale (VAS). Results: The lumbar spinal block was successful in 18 patients, the other 2 showed no greater change with treatment. Conclusions: The lumbar spinal block can reduce pain and improve the functional state of patients with post-laminectomy syndrome, allowing a better quality of life.

KEYWORDS: Laminectomy ; neuromuscular blockade.

\section{INTRODUCCIÓN}

La forma habitual por la que se conoce este proceso en la Literatura en español es "Síndrome de la Cirugía Fallida de la Espalda". Se corresponde con la traducción de la denominación en lengua inglesa: "Failed Back Surgery Syndrome" (1), o síndrome de la Cirugía Fallida de la Espalda es conocido también como lumbalgia de origen desconocido persistente o recurrente, con o sin componente radicular, después de una o más intervenciones quirúrgicas de columna lumbar realizada con la intención de tratar un dolor localizado originalmente en la zona afectada North (2) aclara convenientemente esta cuestión al señalar que nos estamos refiriendo a un fallo o debilidad de la espalda, en lugar del componente negativo (para la cirugía) que representa la denominación en español. Según la Asociación Internacional de Estudios de Dolor - IASP, el Síndrome Postlaminectomía consiste en la aparición o persistencia de dolor lumbar y/o radicular en un paciente que ha sufrido una operación en la columna para tratar un dolor de la misma distribución

Servicio de Medicina Física y Rehabilitación, Hospital Nacional Guillermo Almenara Yrigoyen. Lima, Perú.

Asociación Peruana para el Estudio del Dolor. Lima, Perú.

Servicio de Patología del Desarrollo, Programa de Rehabilitación Pediátrica, Dpto. de Medicina Física y Rehabilitación, Hospital

Nacional Guillermo Almenara Yrigoyen. Lima, Perú.

a Médico Asistente; bresidenta 
topográfica. Importancia del problema: se trata de un proceso de gran importancia clínica y económica, por la elevada incidencia del mismo. Actualmente, en los países de nuestro entorno económico se operan cada año entre 50 y 160 pacientes por cada 100000 habitantes (3). La persistencia de dolor significativo tras estas intervenciones podríamos situarlo en un 20 por 100 de los casos, lo que equivaldría (sólo en nuestro país y considerando las posibilidades más optimistas) a unos 40000 casos de síndrome postlaminectomía al año. Los costes económicos son muy importantes, fundamentalmente en absentismo laboral (más del 90 por 100 del total), aunque en los últimos años los costes económicos derivados del tratamiento médico de este problema están creciendo exponencialmente.

\section{Etiología}

El síndrome postlaminectomía tiene varias causas, a veces difíciles de identificar en un caso concreto $(2,4)$ :

1. Selección inapropiada: que significa que el paciente no era candidato a cirugía por falta de indicación.

2. Selección prematura: Indicación de tratamiento quirúrgico, pero no se han aplicado otros tratamientos conservadores.

3. Selección tardia: Pacientes que reciben el tratamiento quirúrgico de su problema de espalda tardiamente, condicionando lesiones neurológicas establecidas.

4. Cirugía incompleta: Persistiendo o apareciendo síntomas radiculares o de inestabilidad segmentaria.

5. Cirugía complicada: evidencia de casos en los que han acaecidocomplicaciones intray postoperatorias. Aunque no siempre las complicaciones de la cirugía lumbar evidencia dolor crónico ulterior.

6. Fibrosis postquirúrgica: es una de las causas más clásicas a las que achacar el dolor persistente. No debe utilizarse como explicación de primera mano, como a menudo se hace. Es bien sabido que prácticamente todos los pacientes operados desarrollan en mayor o menor grado imágenes RMN compatibles con fibrosis en el nivel operado y la mayor parte de ellos están asintomáticos.

7. Una causa que se introduce en la etiología y en realidad no lo es, es la recidiva al mismo o diferente nivel, después de un periodo a veces muy amplio de buena evolución. En este caso estaríamos hablando de un nuevo proceso.

\section{Clínica}

En el Síndrome Postlaminectomía se pueden presentar tres grandes grupos de síntomas:

1. En primer lugar síntomas de afectación radicular, a veces irritativos y otras veces deficitarios, tanto procedentes de una sola raíz como de varias. Bien afectando a grupos musculares y tejidos, bien a órganos internos. Para su reconocimiento es necesaria una adecuada exploración clínica neurológica y frecuentemente su corroboración con estudios neurofisiológicos.

2. Otro grupo de síntomas es el correspondiente a inestabilidad segmentaria lumbar que se manifiesta en forma de lumbalgia mecánica, es decir que se exacerba con la carga (bipedestación y flexoextensión).

3. El tercer grupo es más heterogéneo y corresponde a los síntomas psicológicos y funcionales derivados del eventual beneficio frente a terceros que se sigue con la persistencia de este cuadro doloroso crónico. Éste grupo no tiene características diferentes del cuadro similar que se presenta en otros procesos.

\section{Diagnóstico}

Es fundamentalmente clínico. Los estudios complementarios buscan confirmar y definir el fundamento de los síntomas del paciente y la pertinencia de su tratamiento en una Unidad del Dolor y no en las manos del cirujano de columna que lo ha tratado. Estos estudios deben realizarse antes de llegar a la Unidad del Dolor, aunque es frecuente que no sea así.

1. Las radiografías simples tienen utilidad si están bien hechas (afirmación evidente por sí misma). A veces perdemos posibilidades diagnósticas si transigimos con estudios técnicamente defectuosos. Hemos de buscar dos grandes procesos: osteólisis e inestabilidad segmentaria.

2. Una analítica elemental tal como la VSG o la Proteína C Reactiva puede ser el único parámetro útil en el diagnóstico y/o seguimiento de algunos cuadros dolorosos postquirúrgicos en los que se asocia cierto grado de espondilitis y/o discitis.

3. La EMG y ENG. Pueden ser de gran utilidad sobre todo para objetivar lesiones nerviosas y si las mismas son agudas o crónicas.

4. La Resonancia Magnética Nuclear se ha convertido en uno de los pilares fundamentales en el diagnóstico del Síndrome Postlaminectomía. ${ }^{5}$

El objetivo del presente trabajo fue evaluar el beneficio del bloqueo para espinal lumbar en el tratamiento del Síndrome Post Laminectomía frente a la aplicación de agentes físicos. 


\section{MATERIAL Y MÉTODOS}

Se realizó un estudio randomizado en pacientes con diagnóstico de síndrome postlaminectomía. Cuarenta (40 pacientes) fueron asignados para recibir tratamiento de los cuales 20 recibieron bloqueo para espinal más terapia física y 20 sólo terapia física. Hemos evaluado la intensidad del dolor antes y después de la aplicación de cada terapia mediante la escala visual análoga (EVA) (escala de $0 \mathrm{~cm}$ (no dolor) a 10 $\mathrm{cm}$ (dolor muy severo) y el estado funcional mediante el cuestionario de discapacidad por dolor lumbar de Oswestry de 0 (no discapacidad) a 100 (discapacidad total).

\section{RESULTADOS}

El bloqueo para espinal lumbar fue exitoso en 18 pacientes, los otros 2 no mostraron mayor cambio con el tratamiento. El grupo que recibió bloqueo para espinal lumbar y terapia física tuvo una reducción promedio de $4 \mathrm{~cm}$ en la intensidad del dolor a las 4 semanas de tratamiento, en comparación con una reducción de 1,7 en el grupo que recibió sólo terapia física. $(\mathrm{P}=\mathrm{X})$. Hubo mejoría clínica significativa en el estado funcional; encontrándose una reducción de 30,2 puntos en los pacientes que recibieron terapia física más bloqueo para espinal lumbar, en comparación de 17,7 puntos en los pacientes que recibieron sólo terapia física $(\mathrm{P}=\mathrm{X})$.

No se observaron complicaciones en la aplicación del procedimiento. Todos los pacientes de ambos grupos culminaron la terapia luego de las cuatro semanas.

Posterior a la ramdomización no hubo diferencias iniciales entre los grupos de tratamiento en cuanto las medias de edad, numero de cirugías y las puntuaciones de las evaluaciones EVA y Oswestry (tabla 1).

Tabla 1. Medias de los grupos de estudio.

\begin{tabular}{ccccccc}
\hline & \multicolumn{6}{c}{ Grupos de Tratmiento } \\
& TF+Bloqueo & \multicolumn{1}{c}{ TF } \\
& N & Mean & DE & N & Mean & DE \\
\hline ESCALA VISUAL ANALOGA* & 20 & 7.10 & 2.02 & 20 & 7.10 & 1.41 \\
OSWESTRY & 20 & 47.90 & 23.93 & 20 & 53.30 & 21.69 \\
EDAD* & 20 & 52.60 & 13.06 & 20 & 54.45 & 5.37 \\
$\mathrm{~N}^{\circ}$ CIRUGIAS* & 20 & 1.70 & 1.49 & 20 & 1.25 & .55 \\
\hline
\end{tabular}

a. ${ }^{*}$ NS prueba $t$ student $95 \%$

Tabla 2. Independent samples test

\begin{tabular}{|c|c|c|c|c|c|c|c|c|c|c|}
\hline & & \multicolumn{2}{|c|}{$\begin{array}{l}\text { Levene's Test } \\
\text { for Equality } \\
\text { of Variances }\end{array}$} & \multicolumn{7}{|c|}{ t-test for Equality of Means } \\
\hline & & \multirow[b]{2}{*}{$\mathbf{F}$} & \multirow[b]{2}{*}{ Sig. } & \multirow[b]{2}{*}{$t$} & \multirow[b]{2}{*}{ df } & \multirow[b]{2}{*}{$\begin{array}{c}\text { Sig. } \\
\text { (2-tailed) }\end{array}$} & \multirow[b]{2}{*}{$\begin{array}{c}\text { Mean } \\
\text { Difference }\end{array}$} & \multirow[b]{2}{*}{$\begin{array}{l}\text { Std. Error } \\
\text { Difference }\end{array}$} & \multicolumn{2}{|c|}{$\begin{array}{l}95 \% \text { Confidence } \\
\text { Interval of the } \\
\text { Difference }\end{array}$} \\
\hline & & & & & & & & & Lower & Upper \\
\hline $\begin{array}{c}\text { Escala } \\
\text { visual analc }\end{array}$ & $\begin{array}{l}\text { Equal variance } \\
\text { assumed } \\
\text { Equal variance } \\
\text { not assumed }\end{array}$ & 5.256 & .028 & $\begin{array}{l}5.438 \\
5.510\end{array}$ & $\begin{array}{r}37 \\
30.777\end{array}$ & $\begin{array}{l}.000 \\
.000\end{array}$ & $\begin{array}{l}2.63 \\
2.63\end{array}$ & $\begin{array}{l}.48 \\
.48\end{array}$ & $\begin{array}{l}1.65 \\
1.65\end{array}$ & $\begin{array}{l}3.61 \\
3.61\end{array}$ \\
\hline Oswestry & $\begin{array}{l}\text { Equal variance } \\
\text { assumed } \\
\text { Equal variance } \\
\text { not assumed }\end{array}$ & 19.677 & .000 & $\begin{array}{l}5.364 \\
5.364\end{array}$ & $\begin{array}{r}38 \\
22.856\end{array}$ & $\begin{array}{l}.000 \\
.000\end{array}$ & $\begin{array}{l}25.20 \\
25.20\end{array}$ & $\begin{array}{l}4.70 \\
4.70\end{array}$ & $\begin{array}{l}15.69 \\
15.48\end{array}$ & $\begin{array}{l}34.71 \\
34.92\end{array}$ \\
\hline
\end{tabular}


Hay suficiente evidencia estadística a un nivel de significancia de 0.05 para afirmar que las medias de las valoraciones de las pruebas Escala Visual Análoga y Oswestry entre los grupos de tratamiento al dolor difieren significativamente $(\mathrm{p}<0.0000, \mathrm{~T}$ student $)$ (tabla 2).

\section{CONCLUSIONES}

El bloqueo para espinal lumbar puede reducir el dolor y mejorar el estado funcional de los pacientes con síndrome post-laminectomía, permitiendo una mejor calidad de vida.

\section{Correspondencia:}

Juana Arias Ramirez

Correo electrónico:giordira@yahoo.com

\section{REFERENCIAS BIBLIOGRÁFICAS-}

1. Del Pozo C. Dolor por cirugía fallida de la espalda. En: Torres L. Medicina del dolor. Barcelona: Masson SA; 2007. p. 299-308.

2. North RB. Chronic low back pain and failed back surgery syndrome. En: North R. Neurosurgical management of pain. Nueva York: Springer-Verlag; 2007. p. 340-9.

3. Cherkin DC, Deyo RA, Loesser JD, Bush T, Waddell G. An international comparison of back surgery rates. Spine. 2004; 19: 1201-6.
4. Yong K. The failed back syndrome: personal opinions and experiences. Chir Org Mov. 2004; 79: 131-2.

5. Yukawa Y, Kato F, Nakamura S. Serial gadolinium enhanced MR imaging after lumbar disc resection: observation of the effected root. J Spinal Disord. 2007; 10: 404-9.

6. Walsh K. Low backs pain in eight areas of Britain. J Epidemiol Comm Health. 2002; 46: 227-30.

7. Young RB. Preoperative and intraoperative predictors of lumbosacral surgery outcome: a literature review. Pain Reviews. 2006; 3: 203-19.

8. Brotchi J, Pirotte B, de Witte O, Levivier M. Prevention of epidural fibrosis in a prospective series of 100 primary lumbo-sacral discectomy patients: follow-up and assessment at repoperation. Neurol Res. 2002; 21 (supl. 1): S47-50.

9. Torres LM, Calderon E, Rey RM. Fentanilo transdermico (Durogesic): características farmacológicas y aplicación clínica. Rev Soc Esp Dolor. 2009; 6: 121-31.

10. Fenollosa P, Salazar H, Canós MA, Pallarés J. Eficacia del TENS a largo plazo en el dolor crónico no maligno. Rev Soc Esp Dolor. 2009; 6: 351-9.

11. Fredman B, Zohar E, Nun MB, Iracji R, Jedeikin R, Gepstein R. The effect of repeated epidural sympathetic nerve block on Failed back Surgery syndrome associated chronic low back pain. J Clin Anesth. 2009; 11: 46-51.

12. López JA. Síndrome Postlaminectomía. Actualizaciones en Dolor. 2000; 1: 51-64. 